\title{
COMMUNICATIONS FOR UAS INTEGRATION IN THE NAS PHASE 2 - SATELLITE COMMUNICATIONS AND TERRESTRIAL EXTENSION
}

\author{
James H. Griner and Robert J. Kerczewski, NASA Glenn Research Center, Cleveland, Ohio
}

\begin{abstract}
In order to provide for the safe integration of unmanned aircraft systems (UAS) into the National Airspace System, the command and control communications link connecting the ground-based pilot with the unmanned aircraft must be highly reliable and robust, with national and international standards to enable interoperability and certification. Both line-of-sight (LOS) links using terrestrial-based communications and beyond-line-of-sight (BLOS) links using satellite communications, supported by national and international standards, are required for integrated UAS operations. The National Aeronautics and Space Administration (NASA) has undertaken an extensive technology development and test program in order to provide the required technical data needed to enable C2 standards development. NASA's UAS Integration in the National Airspace System (NAS), or UAS in the NAS Project, included as a major element the Command and Control Communications (C2) Subproject, based at NASA's Glenn Research Center. The successful first phase of the C2 Subproject, completed during 2012-2016, focused primarily on line-of-sight communications. Accomplishments included air-ground channel propagation characterization and modeling; CNPC prototype radio development; CNPC radio flight testing; satellite communications spectrum study and interference analysis; and development of C2 LOS communications standards development. The second phase of the C2 Subproject will focus primarily on beyond-line-of-sight communications, although a follow-on activity for terrestrial LOS communications, known as Terrestrial Extension, is also included. In addition to the terrestrial element, Phase 2 also includes technology development and testing activities for Ka-Band BLOS C2 Satellite Communications; Ku-Band BLOS C2 Satellite Communications; Ku-Band Interference and Propagation; and C-Band Satellite Communications. This paper will provide brief overviews of the C2 Subproject and its Phase I accomplishments, followed by a description of the plans for the C2 Subproject Phase 2.
\end{abstract}

\section{Introduction}

To enable unmanned aircraft system (UAS) operations to progress from occasional missions requiring specific authorization to routine access to the airspace supporting large numbers of UAS operating whenever and wherever needed, the development and validation of key systems supporting safe UAS and airspace operations is required. In particular, the command and control (also referred to as control and non-payload communications) link connecting the ground-based pilot with the unmanned aircraft must be highly reliable and robust. Both line-of-sight (LOS) links using terrestrial-based communications and beyondline-of-sight (BLOS) links using satellite communications are required. The process of developing national and international standards needed to support C2 link development and certification occurs under the auspices of RTCA in the United States, EUROCAE in Europe, and the International Civil Aviation Organization (ICAO). The development of standards requires the support of technical data characterizing the systems to be standardized and their operating environment.

NASA has been executing the UAS Integration in the National Airspace System (NAS) Project since 2012 to remove technical barriers to UAS integration in such areas as sense and avoid, separation assurance, human/systems integration and C2 communications. During Phase 1 of the C2 Subproject, from 2012 to 2016, the focus was on radio line-of-sight (LOS) C2 links using terrestrialbased communications. Phase 2 of the C2 Subproject, from 2017 to 2020, will primarily address beyond radio line-of-sight (BLOS) C2 links, along with some additional LOS C2 Terrestrial Extension link testing. The following sections provide an overview of the scope and objectives of the C2 Subproject, an overview of key Phase I results, and a description of the activities planned and underway for Phase 2. 


\section{Overall UAS in the NAS C2 Subproject Objectives}

The C2 Subproject, executed at NASA's Glenn Research Center (GRC), was established to overcome technical barriers to the integration of UAS into nonsegregated airspace by enabling the establishment of standards and technical recommendations for UAS C2 communications. In collaboration with other government agencies and industry partners, four primary objectives were addressed.

- Frequency spectrum allocations for both LOS and BLOS UAS C2.

Analysis, sharing studies, and advocacy to support the establishment of spectrum allocations, and define technical requirements of operational spectrum use for UAS.

- Develop and validate UAS control communications Minimum Operational Performance Standards (MOPS).
Through technology collaborative prototype development, laboratory and flight testing, develop technical foundations for the development of MOPS by RTCA Special Committee 228 and assure compliance with national and international regulations, standards, and practices.

- Develop security recommendations for civil UAS control communications.

Establish requirements for security of the UAS C2 system and develop, test and validate technical recommendations for UAS C2 system security.

- Support recommendations for integration of UAS in the NAS.

Through flight testing, C2 system modeling and simulation, and analysis develop and validate technical recommendations for integration of UAS into the airspace.

\section{C2 Subproject Phase 1 Overview}

In Phase 1 of the C2 Subproject, the focus was on development of the technical data for supporting the completion of MOPS for C2 terrestrial systems for LOS C2 communications. This work included airground channel propagation studies, prototype radio development and testing, and radiofrequency spectrum analysis and sharing studies [1].

\section{Air-Ground Channel Propagation}

Propagation studies and modeling of the airground channel were based on a series of channel sounding flight campaigns [2]. Two bands, for which terrestrial C2 LOS links are possible using aviation safety communications spectrum, were studies: LBand (960-1164 MHz) and C-Band (5030-5091 $\mathrm{MHz}$ ). Spread spectrum signals were transmitted from a self-contained, mobile ground station platform equipped with radio frequency test electronics and a 60-foot tall extendable antenna mast. Test signals are received and recorded in-flight on-board a jet research aircraft equipped with specialized signal detection equipment, with two receive antennas in each of the two bands tested, as shown in Figure 1.

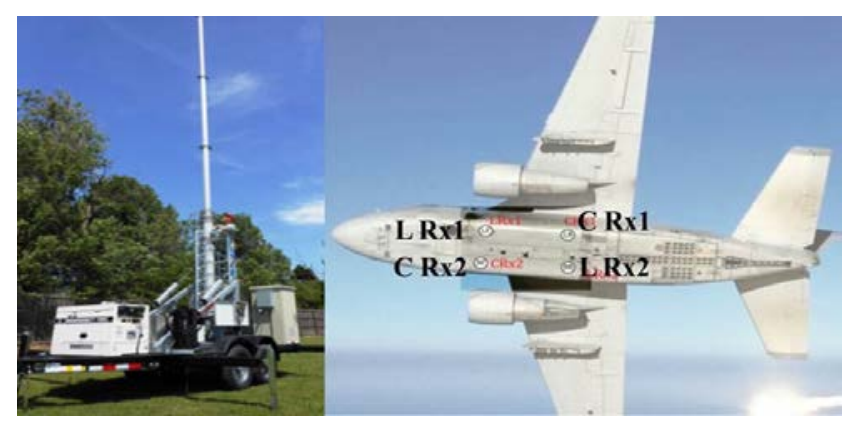

Figure 1. Ground Station and Antenna Tower on Transportable Trailer S Trailer and the NASA GRC S-3B Viking Test Aircraft with Receive Antenna Locations

As the air-ground channel can vary significantly across different types of terrain, several terrain types were studied: mountainous, hilly, flat terrain, nearurban and suburban and over salt and fresh water. The resulting recorded data provided channel impulse responses as a function of link distance. The received LOS signal component, surface reflection component, and multipath components (MPC) are derived from this data enabling computation of power delay profiles. Figure 2 shows an example for mountainous terrain measurements where mountains at a distance of $10 \mathrm{~km}$ create strong multipath components. 

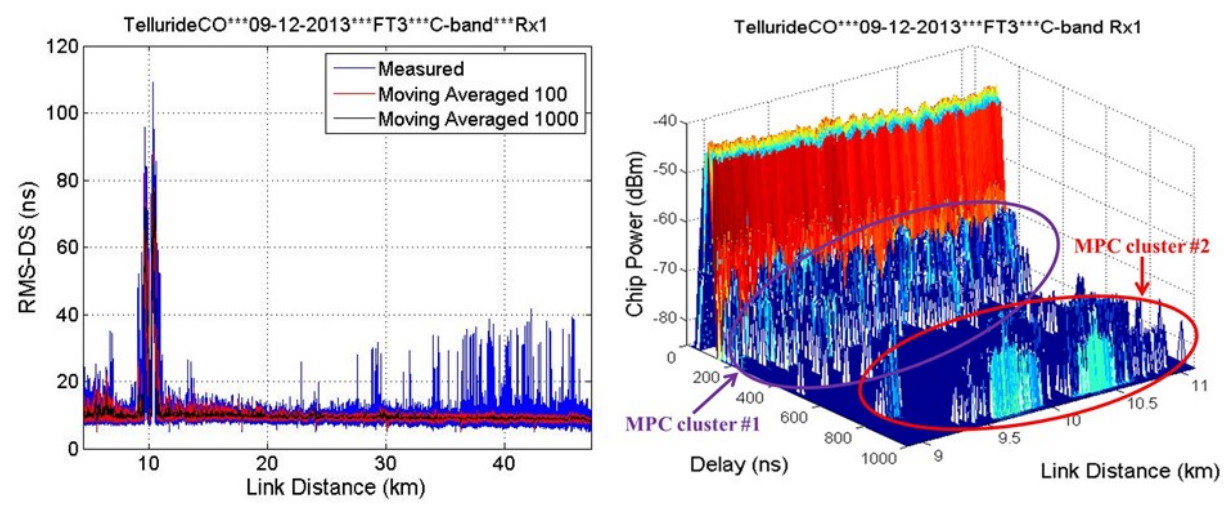

Figure 2. C-Band Multipath Delay Spread Results for Mountainous Terrain: (left) RMS-Delay Spread vs. Link Distance, and (right) Sequence of Power Delay Profiles for RMS-Delay Spread “Bump” Near 10 km

Path loss models and small scale fading parameters have been derived for the terrain types studied. The curved-earth two ray model (including spherical earth divergence and sea-surface roughness effects) was found to fit the measured path loss data better than the flat-earth two-ray and free space models, although the free space model predicts the mean value well for these link distances. Air frame shadowing models have been developed that, although specific to the S3-B aircraft used in the channel measurement campaign, nevertheless provide significant insight into shadowing effects.

\section{C2 Radio Development and Testing}

To provide C2 LOS terrestrial communications system performance data for MOPS development, prototype C2 radios were developed, bench-tested and flight tested to demonstrate data transfer performance, coverage distance and limits, signal loss and recovery and handoffs between ground stations in a variety of terrain types. C2 systems and networks modeling and simulation added to results that supported the MOIPS development effort.

NASA partnered with Rockwell Collins through a shared resource cooperative agreement to demonstrate and support the further development the UAS C2 LOS system. Five generations of prototype $\mathrm{C} 2$ radios, both ground and airborne versions, were used to validate the MOPS. Radios operated in allocated UAS radio frequency spectrum in the 960 $977 \mathrm{MHz}$ and $5030-5091 \mathrm{MHz}$ bands. A complete CNPC system, including interfacing to a ground based pilot station, transmission of CNPC data to/from more than one ground station, and onboard reception and transmission of CNPC data on more than one UA was tested. Testing included hand-off, coverage limits, signal loss and recovery in mountainous, desert, hilly, urban, and over water environments. Figure 3 provides an example of test flight tracks.

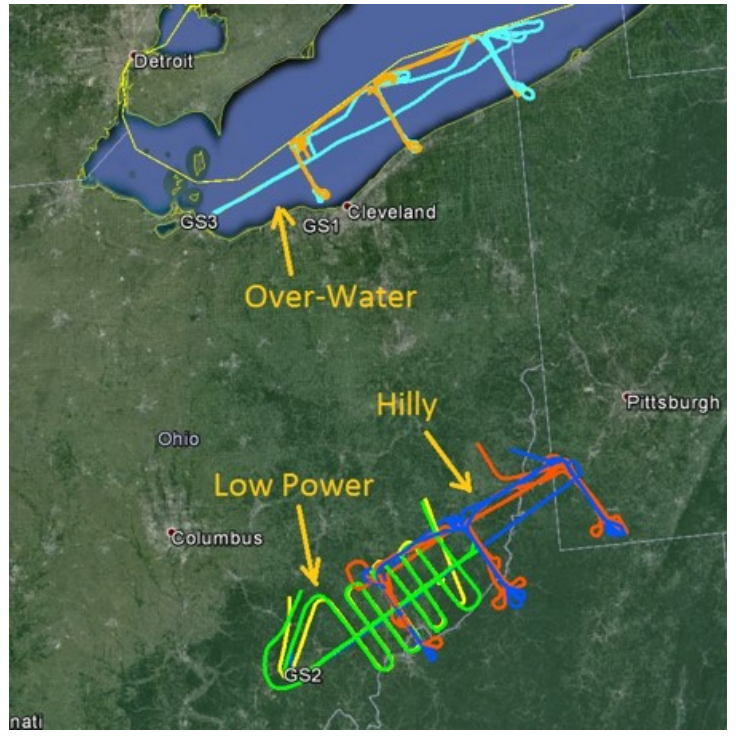

Figure 3. C2 Radio Flight Test Tracks for Several Flights in Northern and Southern Ohio (Image (C)2016 GoogleEarth [7]).

In total, there were over 65 mission flights flown, resulting in more than 200 hours of flight data collected at 12 locations throughout the United States. 


\section{UAS C2 Spectrum}

Spectrum sharing studies and related analyses performed during this period looked at both LOS and BLOS UAS C2 spectrum allocations. The majority of the work addressed spectrum for BLOS CNPC using satellite communications. In particular, studies supported the 2015 World Radiocommunication Conference (WRC-15) Agenda Item 1.5, which considered "the use of frequency bands allocated to the fixed-satellite service not subject to Appendices 30, 30A and 30B for the control and non-payload communications of unmanned aircraft systems (UAS) in non-segregated airspaces." This agenda item, recognizing that existing satellite networks operating in the fixed satellite service (FSS) in the frequency bands at 14/12 GHz (Ku-Band) and 30/20 GHz (KaBand) have potential spectrum capacity to meet the requirements for UAS BLOS C2, required studies and analysis to determine the ability to operate $\mathrm{C} 2$ BLOS links in these bands. The studies performed under the C2 Subproject investigated sharing between UAS C2 satellite transmitters and terrestrial systems of the Fixed Service having co-primary allocations. Results of these studies are described in [3, 4]. WRC-15 approved Resolution 155, making allocations in both $\mathrm{Ku}-$ and Ka-Bands. These allocations are subject to final approval at the next two WRCs, with several technical and regulatory issues to be finalized.

\section{Standards Development}

Technical data developed by the C2 Subproject supported the development of RTCA Document DO362, Command and Control (C2) Data Link Minimum Operational Performance Standards (MOPS) (Terrestrial) developed by RTCA Special Committee 228 (SC-228). Based on the results of laboratory and flight testing, system and network modeling and simulation, system security analysis, spectrum studies and operational analyses, the C2 Subproject contributed directly to the development of major sections of the MOPS document and led the SC-228 subgroups for Security, Verification and Validation.

The following sections and appendices were developed in full or part by the C2 Subproject:

- Equipment Performance Requirements and Test Procedures - Section 2
- Common Characteristics - Section 2.2.1

- MOPS Baseline CNPC Link System

Requirements - Section 2.2.2

- CNPC Link System Manufacturer-Specific Radio Requirements - Section 2.2.3

- Equipment Performance Verification Procedures - Section 2.4

- Security Considerations - Appendix D

- UAS CNPC Link System Operational Capabilities and Implementation Considerations - Appendix F

- Data Rates - Appendix J

- UAS CNPC Link Performance (Based on NASA GRC Flight Test Data) - Appendix K

- Example CNPC Link Budgets - Appendix L

- MOPS Baseline CNPC Link System - Appendix $\mathrm{M}$

- Bench Test Data for the MOPS Baseline CNPC Link System - Appendix N

- Flight Test Data for the MOPS Baseline CNPC Link System - Appendix O

- Compatibility of TACAN Operations and CNPC Operations using L-Band Signals (Based on baseline radio design) - Appendix $P$

- Summary of NASA Air-Ground Channel Measurements and Models - Appendix Q

- CNPC Link Undesired-to-Desired Signal Ratios (Based on NASA GRC Flight Test Data) Appendix R

\section{C2 Subproject Phase 2}

In Phase 2 of the C2 Subproject, planned for 2017-2020, the focus will be BLOS C2 links. Similar to the LOS Phase, the development, bench-testing and flight-testing of C2 radios for the satellite C2 link will be a key element. Using portions of FSS spectrum in Ku-Band and Ka-Band allocated at WRC-15, performance validation and development of technical data to support MOPS development will be undertaken. Characterization of the air-space and ground-space channels will be performed. Importantly for the completion of the spectrum allocations, characterization of the interference environment between the UAS radio and terrestrial systems that operate in these bands will be performed - both terrestrial-to-UAS interference and UAS-toterrestrial system interference will be studied. In addition to the work on the BLOS C2 satellite communication links, there will be an activity to 
extend the C2 LOS terrestrial link MOPS to include lower altitude operations, referred to as Terrestrial Extension.

\section{C2 Subproject Phase 2 - Terrestrial Extension}

RTCA SC-228 has determined that extension of the C2 Terrestrial MOPS (i.e. DO-362) is needed to cover the operational environment of smaller, lower altitude, higher traffic density mid-size UAS operations. The terrestrial extension activity will develop an appropriate terrestrial link between the UAS and the Ground Control Station that supports the required performance of the unmanned aircraft in the NAS, ensures that the pilot always maintains a threshold level of control of the aircraft, and is robust to both environmental or technological issues for this operational environment.

The Terrestrial Extension effort will include a technology assessment activity to consider communication signal waveform and access considerations for the smaller, lower altitude, higher traffic density mid-size UAS operational environment. The conclusions from the technology assessment will drive the development of additional generations of prototype C2 terrestrial radio system consisting of both ground and airborne equipment. CNPC terrestrial radio system, enabling laboratory and flight tests in a relevant flight environment to be conducted. The technical data gathered and analyzed from this test program will be used to support the extension of the current RTCA SC-228 C2 Terrestrial MOPS to cover C2 for lower flight level and higher density UAS operations.

Figure 4 provides a comparison of flight testing in Phase 1 vs. the planned flight testing for Phase 2. Phase 1 included the air-ground channel propagation flight testing performed at an altitude of $2000 \mathrm{ft}$., the primary radio flight tests performed at $17,500 \mathrm{ft}$. and some additional testing for airport arrival and departure environment at $1000 \mathrm{ft}$. Testing for Phase 2 will at altitudes as low as $500 \mathrm{ft}$.

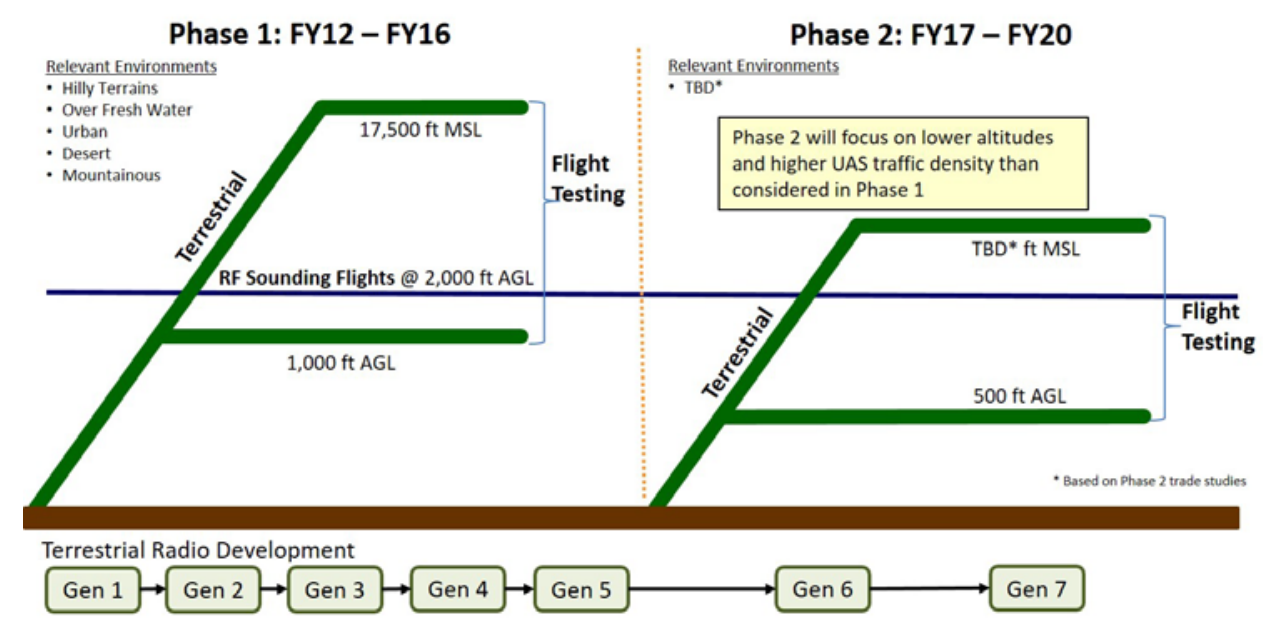

Figure 4. C2 Radio Flight Test Environment - Phase 1 and Phase 2

\section{C2 Subproject Phase 2 - Ka-Band Satellite Communications}

With the adoption of Resolution 155, WRC-15 has established allocations in the Fixed Satellite Service bands in which UAS C2 satellite communications can operate. The Resolution requires additional actions at the next two future WRCs, but RTCA SC-228 is proceeding to develop
MOPS for the BLOS C2 communications systems that would operate in the Ku-Band and Ka-Band allocations defined by Resolution 155, as well as in a previous C-Band allocation to the Aeronautical Mobile Satellite (Route) Service (AMS(R)S) which is available for BLOS C2. In addition, ICAO is developing Standards and Recommended Practices (SARPs) for BLOS C2 communications for these bands. 
The objective of both the Ku-Band and KaBand C2 satellite communications efforts is to develop an appropriate satellite communicationsbased link between the UAS and the ground control station (GCS) that supports the required performance of the unmanned aircraft in the NAS, ensures that the pilot always maintains a threshold level of control of the aircraft, and is robust to both environmental or technological issues. As with LOS terrestrial C2 communications in Phase 1 and 2 of the C2 subproject, performance validation and development of technical data to support BLOS C2 satellite communications MOPS development will be undertaken for BLOS satellite communications. To obtain this data, flight testing of the satellite systems in a relevant environment will be performed, including an aircraft equipped with a satellite communications terminal and the groundbased satellite access equipment. Figure 5 describes the elements of this system required to perform endto-end communications latency testing, as well as link performance vs. transmit power, data rate, modulation and coding, multiple access scheme, and other parameters.

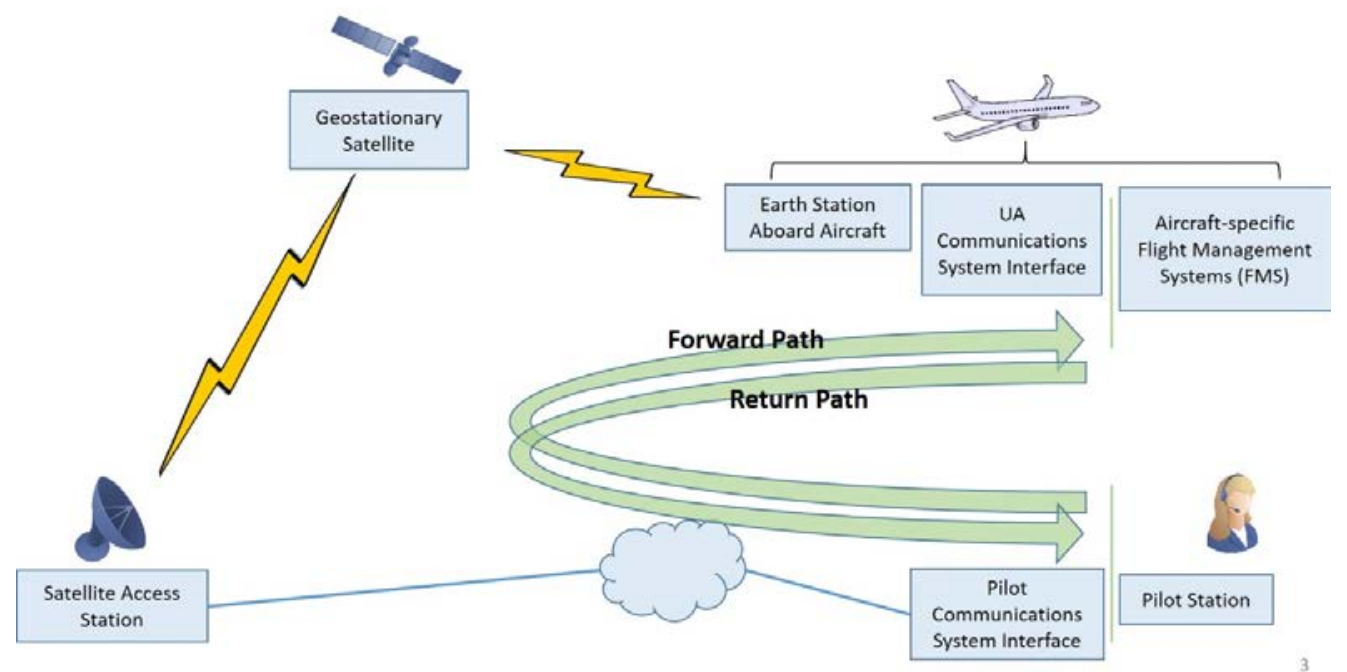

Figure 5. UAS BLOS C2 Satellite Communications Latency Path

In February 2017, NASA GRC entered into a cooperative agreement with Honeywell International, Inc. to address flight testing of a KaBand BLOS satellite communications link. This agreement will enable use of the Inmarsat Global Express network based on Inmarsat's I-5 Ka-Band satellite series [5]. For the airborne and terrestrial segments, Honeywell's JetWave high-speed satellite communications hardware will be employed [6].

Ka-Band flight testing will make use of frequencies defined in WRC-15 Resolution 155: 29.5-30.0 GHz for earth-to-space and 19.7-20.2 $\mathrm{GHz}$ for space-to-earth. Testing will occur at enroute flight altitudes with simulated UAS automated operations. Two types of Ka-Band aircraft satellite communications will be tested: fuselage-mounted phased array and tail-mounted mechanically steerable parabolic reflector. A NASA GRC test Aircraft will be used for the flight testing. Detailed planning of the Ka-Band flight testing was initiated in March 2017.

\section{C2 Subproject Phase 2 - Ku-Band Satellite Communications}

The flight testing described in the preceding section for BLOS C2 satellite communications in Ka-Band will be duplicated in the Ku-Band frequencies defined in Resolution 155: 14.0-14.47 $\mathrm{GHz}$ for earth-to-space, and 10.95-11.2 GHz, 11.45-11.7 GHz, 11.7-12.2 GHz space-to-earth bands applicable globally or in ITU Region 2. A cooperative agreement partner was not available for Ku-Band testing, therefore NASA GRC will 
develop the required aircraft and ground terminal equipment and identify a suitable $\mathrm{Ku}$-Band satellite network for the flight testing.

\section{C2 Subproject Phase 2 - Ku-Band Interference and Propagation}

Although the propagation characteristics of the earth-space channel in Ku-Band and Ka-Band have been extensively studied and are well understood, the characteristics of the air-ground channel in these bands has not been sufficiently characterized. The air-ground channel is particularly important for $\mathrm{Ku}-$ Band as co-primary allocations to the Fixed Service (FS) exist in same bands allocated to for BLOS C2 satellite communications by WRC-15 Resolution 155. Further, Resolution 155 directs that power flux density (pfd) limits for UAS C2 satellite communications terminal transmitters be determined and agreed at WRC-19. The pfd limits must be sufficient to protect FS station receivers from interference originating from C2 transmitters in accordance with established FS protection criteria. Therefore, knowledge of the characteristics of the air-ground channel will be of value in understanding potential interference from the UAS BLOSC2 link in Ku-Band.

To obtain this information, Phase 2 of the C2 Subproject will include a flight test campaign to characterize the Ku-Band air-ground channel. A
NASA aircraft will be equipped with a transmitter operating in the 14.0-14.47 GHz band with resulting emission reaching the ground measured with appropriate ground receiving and measuring equipment. Figure 6 depicts the geometry of this measurement, with the ground receiver measuring LOS, ground reflection and multipath components resulting from the airborne transmission.

The initial experiment design consists of the following components: An omni-directional transmit antenna will be mounted on the bottom of the test aircraft fuselage to provide the transmitted signal. The ground receiver will include two antennas- a $60 \mathrm{~cm}$ diameter parabolic reflector with a 2.5 degree beamwidth and gain of $36 \mathrm{dBi}$, and a horn antenna with 20 degree beamdwidth and 15 $\mathrm{dBi}$ of gain. The $60 \mathrm{~cm}$ reflector antenna is similar to typical Fixed Service station installations and will allow the observance of received signal strength and duration that may be observed in operational scenarios, while the broad beamwidth horn antenna will allow signal measurements for longer durations to obtain more signal propagation data. The ground receivers will be mounted at a height of 10 meters, with antennas pointed at an elevation angle of 5 degrees. Both vertical and horizontal polarizations will be tested.

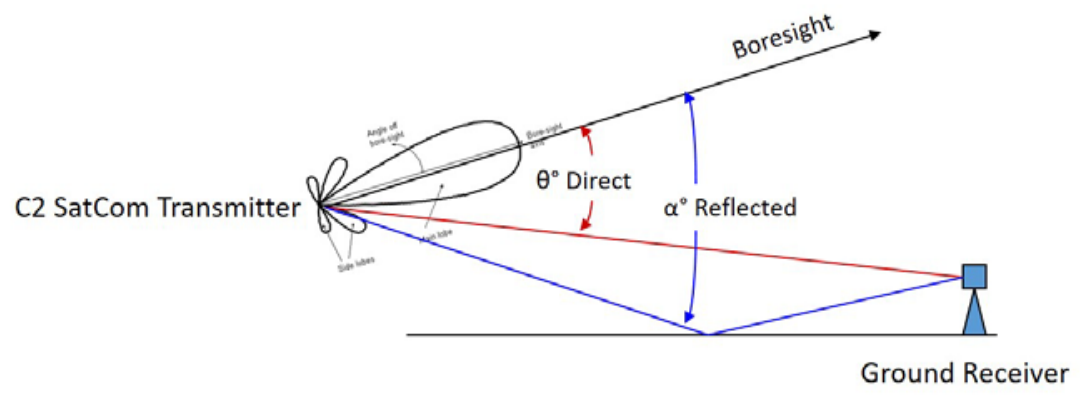

Figure 6. Measurement of the Ku-Band Air-Ground Channel

\section{C2 Subproject Phase 2 - C-Band Satellite Communications}

An AMS(R)S allocation in C-Band covers the 5.03-5.091 GHz band and would also be suitable for BLOS C2 satellite communications. Therefore, this band will also be studied to provide technical data for MOPS development. However, the lack of an existing satellite operating in this band makes it impossible to conduct a realistic flight experiment.

The C-Band activity will consist of the definition of a BLOS C2 satellite communication 
system link to a design level that supports the performance requirements. The approach will be to develop initial design parameters of airborne and ground station equipment, and to develop a preliminary payload design, in order to assess the feasibility of an operational C-Band satellite-based C2 system. Technology assessments will provide design guidance and also provide technical data for MOPS development.

\section{Summary}

NASA's UAS in the NAS Project's C2 Subproject has entered Phase 2. Phase 1 (20122016) emphasized LOS C2 terrestrial communications and the development of technical data for supporting the completion of C2 Terrestrial MOPS by RTCA SC-228. Major activities brought to a successful completion in Phase 1 include: characterization of the air-ground channel in LBand and C-Band and development of air-ground channel models; development of a C2 prototype radio and laboratory and flight testing of the radio system, including system and network modeling and simulation and system security analysis; analysis and sharing studies supporting CS BLOS spectrum allocation; and direct contributions to substantial portions of the MOSP document.

Phase 2 (2017-2020) emphasizes BLOS C2 satellite communications, with an additional terrestrial extension element. The terrestrial extension of the C2 Terrestrial MOPS (i.e. DO-362) is needed to cover the operational environment of smaller, lower altitude, higher traffic density midsize UAS operations and will be accomplished through technology assessment, additional C2 terrestrial development, and laboratory and flight testing. Phase 2 will include the following $\mathrm{C} 2$ satellite communications efforts: Ka-Band C2 satellite communication system development and testing, through a cooperative agreement with Honeywell International Inc.; Ku-Band C2 satellite communication system development and testing performed through an in-house effort at NASA GRC; Ku-Band interference and propagation testing to characterize the air-ground channel at Ku-Band; and C-Band C2 satellite communications studies to develop a C-Band C2 satellite system design to assess feasibility and develop technical performance information. As with Phase 1, technical data developed for the C2 link will support SC-228 development of BLOS C2 satellite communications MOPS.

\section{References}

[1] Kerczewski, R. J., J. H. Griner, W. D. Bishop, D. W. Matolak, and J. D. Wilson, March 2017, Progress on the Development of the UAS C2 Link and Supporting Spectrum - from LOS to BLOS, 2017 IEEE Aerospace Conference.

[2] RTCA Document DO-362, Appendix Q.

[3] Kerczewski, R. J., J. D. Wilson, and W. D. Bishop, March 2016, UAS CNPC Satellite Link Performance - Sharing Spectrum with Terrestrial Systems, 2016 IEEE Aerospace Conference.

[4] Kerczewski, R. J., J. D. Wilson, and W. D. Bishop, October 2016, Satellite Communications for Unmanned Aircraft C2 Links - C-Band, KuBand and Ka-Band, 22nd $\mathrm{Ka}$ and Broadband Communications Conference.

[5] Inmarsat Global Express

http://www.inmarsat.com/service/global-xpress/.

[6] Honeywell JetWave

https://aerospace.honeywell.com/en/products/safety -and-connectivity/jetwave-high-speed-satellitecommunications-hardware.

[7] Map data (2016 Google. Google, Inc., 1600 Amphitheatre Parkway, Mountain View, CA 94043, http://www.google/com/earth/.

\section{Integrated Communications Navigation and Surveillance (ICNS) Conference}

April 18-20, 2017 